\title{
Helicobacter Pylori Infection in Waste Pickers: A Case Control Seroprevalence Study
}

\author{
Cosme Alvarado-Esquivel ${ }^{\mathrm{a}, \mathrm{b}}$
}

\begin{abstract}
Background: The epidemiology of Helicobacter pylori (H. pylori) infection in waste pickers had not been previously studied. This study aims to determine the association of $H$. pylori seropositivity and waste picking activity; and to determine socio-demographic, clinical, work, and behavioral characteristics associated with $H$. $p y$ lori seropositivity in waste pickers.
\end{abstract}

Methods: Through a case-control study design, we examined 90 waste pickers and 90 age- and gender-matched control subjects for the presence of anti-H. pylori IgG antibodies using enzyme-linked immunoassays. Seroprevalence association with socio-demographic, clinical, work and behavioral characteristics of the waste pickers were also investigated.

Results: Antibodies to H. pylori were found in $60(66.7 \%)$ of the 90 waste pickers and in $51(56.7 \%)$ of the 90 controls $(\mathrm{P}=0.16)$. Stratification by age showed that waste pickers aged $14-30$ years old had significantly higher frequency of $H$. pylori infection than controls of the same age group $(56.5 \%$ versus $35.6 \%$, respectively; $\mathrm{P}=$ 0.04). The seroprevalence of $H$. pylori infection was not influenced by gender, age, educational level, socioeconomic status, residence, or housing conditions of waste pickers. The presence of underlying diseases and the frequency of gastritis were similar among $H$. pylori positive and $H$. pylori negative waste pickers. Logistic regression analysis showed that the duration (years) in the waste picking activity was positively associated with $H$. pylori exposure $(\mathrm{OR}=2.76$; 95\% CI: $1.22-6.25 ; \mathrm{P}=0.01)$. In contrast, consumption of alcohol was negatively associated with $H$. pylori exposure $(\mathrm{OR}=0.27 ; 95 \%$ CI: $0.09-0.78 ; \mathrm{P}=0.01)$. Other work or behavioral characteristics of waste pickers including washing hands before eating, eating

Manuscript accepted for publication September 26, 2013

\footnotetext{
${ }^{a}$ Biomedical Research Laboratory, Faculty of Medicine and Nutrition, Juarez University of Durango State, Avenida Universidad S/N, 34000 Durango, Dgo, Mexico

${ }^{\mathrm{b}}$ Corresponding author: Cosme Alvarado-Esquivel, Biomedical Research Laboratory, Faculty of Medicine and Nutricion, Avenida Universidad S/N, 34000 Durango, Dgo, Mexico.

Email: alvaradocosme@yahoo.com
}

doi: http://dx.doi.org/10.4021/gr578e from the garbage, animal contacts, consumption of unpasteurized milk, unwashed raw vegetables, fruits, or untreated water, and contact with soil were not associated with $H$. pylori exposure.

Conclusions: This is the first report on the seroprevalence of $H$. pylori infection among waste pickers and the factors contributing to such exposure. Results warrant for further research on the potential role of contact with garbage for $H$. pylori infection.

Keywords: Helicobacter pylori; Seroprevalence; Waste pickers; Epidemiology; Mexico

\section{Introduction}

Helicobacter pylori are microaerophilic Gram-negative bacterium currently infecting about one-half of the world's population $[1,2]$. Infections with $H$. pylori may be asymptomatic or may lead to gastric disease including chronic gastritis, peptic ulcer, gastric mucosa-associated lymphoid tissue lymphoma, and gastric cancer [1-4]. Transmission of $H$. pylori might occur from person-to-person [5], through oral-oral or oral-fecal routes [6], or consumption of contaminated water $[6,7]$. Epidemiological studies indicate that prevalence of H. pylori infection varies substantially among countries and prevalence is mainly influenced by country development [8], geographical region, migration pattern and ethnicity $[6,9]$.

There is a lack of information about infections of $H$. $p y$ lori in waste pickers. Some epidemiological characteristics in waste pickers are relevant for acquiring infections with $H$. pylori. Firstly, the oral-oral and oral-fecal routes of $H$. pylori infection may occur in waste pickers. Garbage may contain human excrement, saliva and other fluids, therefore, waste pickers handle potentially contaminated garbage, and even a number of them eat from the garbage. Secondly, many waste pickers drink untreated water. Thirdly, the person-to-person route of infection with $H$. pylori might occur in waste pickers because they have poor living conditions including poor housing where a number of persons cohabitate in a single room. This case-control study aimed to determine the seroprevalence of anti-H. pylori antibodies in waste pickers in Du- 
Table 1. Socio-demographic Characteristics of Waste Pickers and Seroprevalence of H. pylori Infection

\begin{tabular}{|c|c|c|c|c|}
\hline \multirow{2}{*}{ Characteristic } & \multirow{2}{*}{$\begin{array}{l}\text { No. of } \\
\text { subjects } \\
\text { tested* }\end{array}$} & \multicolumn{2}{|c|}{ Prevalence of $\boldsymbol{H}$. pylori infection } & \multirow{2}{*}{ Pvaluc } \\
\hline & & No. & $\%$ & \\
\hline \multicolumn{5}{|l|}{ Gender } \\
\hline Male & 34 & 22 & 64.7 & \multirow[t]{2}{*}{0.75} \\
\hline Female & 56 & 38 & 67.9 & \\
\hline \multicolumn{5}{|l|}{ Age groups (years) } \\
\hline 30 or less & 46 & 26 & 56.5 & \multirow[t]{3}{*}{0.11} \\
\hline $31-50$ & 22 & 16 & 72.7 & \\
\hline$>50$ & 21 & 17 & 80.0 & \\
\hline \multicolumn{5}{|l|}{ Residence place } \\
\hline Durango State & 88 & 58 & 65.9 & \multirow[t]{2}{*}{0.55} \\
\hline Other Mexican State & 2 & 2 & 100.0 & \\
\hline \multicolumn{5}{|l|}{ Residence area } \\
\hline Urban & 83 & 55 & 66.3 & \multirow[t]{2}{*}{0.35} \\
\hline Suburban or rural & 6 & 5 & 83.3 & \\
\hline \multicolumn{5}{|l|}{ Educational level } \\
\hline No education & 28 & 19 & 67.9 & \multirow[t]{3}{*}{0.80} \\
\hline $1-6$ years & 50 & 34 & 68.0 & \\
\hline More than 6 years & 12 & 7 & 58.3 & \\
\hline \multicolumn{5}{|l|}{ Socioeconomic level } \\
\hline Low & 67 & 44 & 65.7 & \multirow[t]{2}{*}{0.70} \\
\hline Medium & 17 & 12 & 70.6 & \\
\hline
\end{tabular}

${ }^{*}$ Participants with available data.

rango City, Mexico. In addition, we also investigated sociodemographic, clinical, work, and behavioral characteristics associated with $H$. pylori

\section{Materials and Methods}

\section{Study design and study population}

Through an age- and gender- matched case-control study using serum samples from recent Toxoplasma gondii serosurveys $[10,11], 90$ waste pickers and 90 control subjects were compared for the presence of anti-H. pylori IgG antibodies. Inclusion criteria for the waste pickers were: 1) waste pickers in the municipal solid waste transfer station of Durango City, Mexico; 2) aged 14 years and older; 3) any gender; 4) waste picking for at least 3 months; and 5) who accepted to participate in the study. Waste pickers included in the study were 14 - 76 (mean $=36.0 \pm 17.1$ ) year-old, 34 were males and 56 were females. Control subjects matched with waste pickers by age and gender. Controls consisted of 34 males and 56 females with miscellaneous occupations other than waste picking including students, employees, homemakers, and business. The mean age in controls was $35.7 \pm 16.8$ (range: $18-78$ ) year-old and comparable with that in waste pickers $(\mathrm{P}=0.91)$.

\section{Socio-demographic, clinical, work, and behavioral data}

We obtained the characteristics of the participants by using a standardized questionnaire. Socio-demographic data including age, gender, birthplace, residence, educational level, and socioeconomic level was collected from all participants. Work characteristics assessed in waste pickers included num- 
Table 2. Work Characteristics of Waste Pickers and Seroprevalence of $H$. pylori infection

\begin{tabular}{|c|c|c|c|c|}
\hline \multirow{2}{*}{ Characteristic } & \multirow{2}{*}{$\begin{array}{l}\text { No. of } \\
\text { subjects } \\
\text { tested* }\end{array}$} & \multicolumn{2}{|c|}{ Prevalence of $\boldsymbol{H}$. pylori infection } & \multirow{2}{*}{ P value } \\
\hline & & No. & $\%$ & \\
\hline \multicolumn{5}{|l|}{ Years working } \\
\hline Up to 10 years & 47 & 26 & 55.3 & 0.03 \\
\hline 11 to 20 years & 29 & 21 & 72.4 & \\
\hline More than 20 years & 13 & 12 & 92.3 & \\
\hline \multicolumn{5}{|c|}{ Number of visiting waste places } \\
\hline One & 80 & 53 & 66.3 & 0.67 \\
\hline More than 1 & 6 & 4 & 66.7 & \\
\hline \multicolumn{5}{|l|}{ Wear hand gloves } \\
\hline Yes & 16 & 10 & 62.5 & 0.64 \\
\hline No & 73 & 50 & 68.5 & \\
\hline \multicolumn{5}{|l|}{ Wear facemask } \\
\hline Yes & 1 & 1 & 100.0 & 1.00 \\
\hline No & 88 & 59 & 67.0 & \\
\hline \multicolumn{5}{|l|}{ Eating while working } \\
\hline Yes & 51 & 34 & 66.7 & 0.86 \\
\hline No & 38 & 26 & 68.4 & \\
\hline \multicolumn{5}{|c|}{ Eating from the garbage } \\
\hline Yes & 26 & 15 & 57.7 & 0.20 \\
\hline No & 63 & 45 & 71.4 & \\
\hline \multicolumn{5}{|c|}{ Washing hands before eating } \\
\hline Yes & 68 & 45 & 66.2 & 0.65 \\
\hline No & 21 & 15 & 71.4 & \\
\hline \multicolumn{5}{|l|}{ Alcohol consumption } \\
\hline Yes & 29 & 15 & 51.7 & 0.02 \\
\hline No & 60 & 45 & 75.0 & \\
\hline \multicolumn{5}{|l|}{ Injury while working } \\
\hline Yes & 64 & 40 & 62.5 & 0.13 \\
\hline No & 24 & 19 & 79.2 & \\
\hline
\end{tabular}

${ }^{*}$ Participants with available data.

ber of years in the activity, habitual use of safety practices (use of hand gloves and face masks), eating while working, drinking alcohol while waste picking, washing hands before eating, eating from the garbage, and ever had suffered from injuries with sharp material of the garbage. Clinical data explored included the presence of underlying diseases in gener- al and gastric disease in particular. Behavioral data included animal contacts, consumption of unpasteurized milk, unwashed raw vegetables, fruits, or untreated water, frequency of eating out of home (in restaurants or fast food outlets), and contact with soil (gardening or agriculture). Housing conditions were examined by using the Bronfman's criteria [12]. 
Table 3. Results of the multivariate regression analysis.

\begin{tabular}{llll}
\hline Variable & P value & Odds ratio & $\mathbf{9 5 \%}$ confidence interval \\
\hline Cats at home & 0.2 & 1.88 & $0.70-5.05$ \\
Raising animals & 0.68 & 1.23 & $0.44-3.42$ \\
Alcohol consumption & 0.01 & 0.27 & $0.09-0.78$ \\
Years in the activity & 0.01 & 2.76 & $1.22-6.25$ \\
\hline
\end{tabular}

Briefly, we evaluated five variables as follows: number of persons in the house, number of rooms in the house, material of the floor of the house, availability of drinkable water, and form of elimination of excreta.

\section{Serological detection of $\mathrm{H}$. pylori antibodies}

Serum samples from cases and controls were analyzed by qualitative and quantitative methods for detection of antiH. pylori IgG antibodies using a commercially available enzyme-linked immunosorbent assay (ELISA) kit, Anti- $H$. pylori IgG AccuBind ELISA (Monobind Inc, Lake Forest, California). Anti-H. pylori IgG antibody levels were expressed as Units $(\mathrm{U}) / \mathrm{mL}$, and a value higher than $20 \mathrm{U} / \mathrm{mL}$ was considered a positive result. ELISA was performed following the manufacturer's instructions.

\section{Statistical analysis}

Statistical analysis was performed using the Epi Info version 3.5.4 software (Centers for Disease Control and Prevention: http://wwwn.cdc.gov/epiinfo/) and SPSS version 15.0 software (SPSS Inc. Chicago, Illinois). Pearson's chi-square test and the Fisher exact test (when values were less than 5) were used to compare frequencies between groups. Bivariate and multivariate analyses were used to assess the association between waste pickers characteristics and $H$. pylori seropositivity. Variables were included in the multivariate analysis if they had a $\mathrm{P}$ value $\leq 0.20$ in the bivariate analysis. Odd ratio (OR) and 95\% confidence interval (CI) were calculated by multivariate analysis using logistic regression analysis with the Enter method. A P value $<0.05$ was considered as statistically significant.

\section{Ethical considerations}

This study was approved by the Ethical Committee of the Instituto de Seguridad y Servicios Sociales de los Trabajadores del Estado in Durango City. The purpose and procedures of the study were explained to all participants, and a written informed consent was obtained from each participant.

\section{Results}

Of the 90 waste pickers tested, $60(66.7 \%)$ were positive for anti-H. pylori IgG antibodies. While of the 90 controls tested, $51(56.7 \%)$ were positive for anti-H. pylori IgG antibodies. In general, cases and controls had similar seroprevalences of anti-H. pylori IgG antibodies $(\mathrm{P}=0.16)$. However, stratification by age showed the differences in the seroprevalence of $H$. pylori infection in cases and controls as follows. A 56.5 percent $(26 / 46)$ versus $35.6 \%(16 / 45)$ in 14 - 30 years group $(\mathrm{P}=0.04) ; 72.7 \%(16 / 22)$ versus $76 \%(19 / 25)$ in 31 - 50 years group $(\mathrm{P}=0.79)$; and $80.1 \%(17 / 21)$ versus $80 \%$ $(16 / 20)$ in 51 - 78 years group $(\mathrm{P}=0.62)$, respectively. General socio-demographic characteristics of the waste pickers studied shown in Table 1. The seroprevalence of $H$. pylori infection was not influenced by gender, age, residence, educational level, or socioeconomic status of waste pickers. Of the $60 \mathrm{H}$. pylori IgG positive waste pickers, $31(51.7 \%)$ had IgG levels higher than $100 \mathrm{U} / \mathrm{mL}, 11(18.3 \%)$ between 51 to $100 \mathrm{U} / \mathrm{mL}$, and $18(30.0 \%)$ between 21 to $50 \mathrm{U} / \mathrm{mL}$. Anti- $H$. pylori IgG antibody levels were similar in men and women $(\mathrm{P}=0.84)$. None of the housing variables examined including number of persons in the house, number of rooms in the house, material of the floor of the house, availability of drinkable water, and form of elimination of excreta showed any association with $H$. pylori infection.

Of the work characteristics in waste pickers (Table 2), the years of duration in the activity and drinking alcohol while waste picking were positively and negatively associated with $H$. pylori infection, respectively. Other work characteristics including habitual use of safety practices, eating while working, washing hands before eating, eating from the garbage, and ever had suffered from injuries with sharp material of the garbage did not influence the seroprevalence of $H$. pylori infection.

With respect to clinical data, the presence of underlying diseases and the frequency of gastritis were similar among 
H. pylori positive and $H$. pylori negative waste pickers.

Concerning behavioral characteristics, bivariate analysis showed 2 characteristics with a $\mathrm{P}$ value equal to or less than 0.20 including: cats at home $(\mathrm{P}=0.13)$, and raising animals $(\mathrm{P}=0.17)$. Other behavioral characteristics including consumption of unpasteurized milk, unwashed raw vegetables, fruits, or untreated water, frequency of eating out of home and contact with soil showed $\mathrm{P}$ values higher than 0.20 in the bivariate analysis. Multivariate analysis (Table 3) of the work and behavioral characteristics with a $\mathrm{P}$ value equal to or less than 0.20 obtained by bivariate analysis showed that the years of duration in the waste picking activity was positively associated with $H$. pylori exposure $(\mathrm{OR}=2.76 ; 95 \%$ CI: 1.22 - 6.25; $\mathrm{P}=0.01)$. In contrast, consumption of alcohol was negatively associated with $H$. pylori exposure (OR $=0.27 ; 95 \%$ CI: $0.09-0.78 ; \mathrm{P}=0.01)$. No further work or behavioral characteristics of waste pickers associated with H. pylori exposure were found.

\section{Discussion}

In the present study, waste pickers showed a slightly higher (but not statistically significant) seroprevalence of $H$. pylori infection than age- and gender-matched control subjects. The general seroprevalence found in waste pickers is comparable to the mean national seroprevalence $(66 \%)$ reported in Mexico [13]. However, these seroprevalence results cannot be directly compared, since different methods were used in both studies; although the present study used a commercial ELISA kit, a homemade ELISA kit was used for the national survey. In an international context, the rate of $H$. pylori seroprevalence in the waste pickers of Durango is lower than the estimated $80-90 \%$ seroprevalence of $H$. pylori infection in developing countries [8]. In the present study, further analysis by age stratification showed that waste pickers aged 14 - 30 years old had a significantly higher seroprevalence of $H$. pylori infection than controls of the same age group. While in older age groups of waste pickers and controls, the seroprevalence of $H$. pylori infection was comparable. These findings suggest that waste picking may be an important factor for $H$. pylori exposure in young subjects but there might be unidentified behavioral, work, or other type of changes leading to a similar frequency of $H$. pylori exposure in both waste pickers and controls of older ages. Therefore, results suggest that young waste pickers represent a risk group for $H$. pylori infection. Of note, the $56.5 \%$ seroprevalence of $H$. pylori infection found in young ( $\leq 30$ years old) waste pickers is also higher than the $37.9 \%$ seroprevalence reported recently in Mennonites of the same age group in a rural community in Durango State [14]. Remarkably, analysis of waste pickers socio-demographic characteristics showed that seroprevalence of $H$. pylori was not only high among the youngest participants but also did not show a statistically significant increase with age. The former suggests an early exposure to H. pylori in waste pickers, and the latter is not consistent with the typical increase in the frequency of infection with age that has been reported in several studies [5, 9, 14-16]. Other putative socio-demographic factors associated with $H$. pylori infection, including low socioeconomic status [5, 13] and low educational level [13], did not influence seroprevalence rates in waste pickers. Similarly, H. pylori seropositivity was not associated with gender or residence. Concerning housing conditions of waste pickers, seroprevalence of $H$. pylori was not influenced by the number of persons in the house, number of rooms in the house, material of the floor of the house, availability of drinkable water, and form of elimination of excreta. These findings suggest that $H$. pylori exposure might have not occurred at home by drinking untreated water, overcrowding or poor sanitary conditions but probably in the waste transfer station by strong contact with garbage likely contaminated with human excrement and fluids.

Concerning clinical data, the presence of underlying diseases and the frequency of gastritis were similar among $H$. pylori positive and $H$. pylori negative waste pickers. This finding suggests that $H$. pylori are not probably influencing on the health of waste pickers.

With respect to work and behavioral characteristics of waste pickers, multivariate analysis showed that the years of duration in the waste picking activity was positively associated with $H$. pylori exposure (OR $=2.76$; 95\% CI: 1.22 - 6.25; $\mathrm{P}=0.01)$. In contrast, consumption of alcohol was negatively associated with $H$. pylori exposure $(\mathrm{OR}=0.27$; $95 \% \mathrm{CI}: 0.09-0.78 ; \mathrm{P}=0.01)$. No further work or behavioral characteristics of waste pickers associated with $H$. pylori exposure were found. The association of $H$. pylori seropositivity and the years of duration in the waste picking activity further support the increased risk for $H$. pylori infection by waste contact. It is not clear how the waste pickers acquired the $H$. pylori infection but it is likely that they acquired it through frequent contact with garbage contaminated with human excrements and fluids. The negative association of alcohol consumption with $H$. pylori seropositivity found in the current study supports previous observations [17-20] and suggests that alcohol consumption might be protective against $H$. pylori infection. However, the negative association of alcohol consumption and $H$. pylori infection conflicts with observations in other studies. Zhang et al [21] reported a positive association of alcohol consumption with $H$. pylori infection in patients with functional dyspepsia. In contrast, no association of alcohol consumption and $H$. pylori infection have been found in blood donors in Brazil [22], and in patients with abdominal complains in a clinic in Japan [23]. Differences in the characteristics of the studied populations including health status and occupation may explain the differences in the associations.

This is the first report on the seroprevalence of $H$. pylori infection among waste pickers and the factors contributing 
to such exposure. Results warrant for further research on the potential role of contact with garbage for $H$. pylori infection.

\section{References}

1. Ruggiero P. Helicobacter pylori and inflammation. Curr Pharm Des. 2010;16(38):4225-4236.

2. Sachs G, Scott DR, Wen Y. Gastric infection by Helicobacter pylori. Curr Gastroenterol Rep. 2011;13(6):540546.

3. Malfertheiner P. The intriguing relationship of Helicobacter pylori infection and acid secretion in peptic ulcer disease and gastric cancer. Dig Dis. 2011;29(5):459464.

4. Zabaleta J. Multifactorial etiology of gastric cancer. Methods Mol Biol. 2012;863:411-435.

5. Ford AC, Axon AT. Epidemiology of Helicobacter pylori infection and public health implications. Helicobacter. 2010;15(Suppl 1):1-6.

6. Goh KL, Chan WK, Shiota S, Yamaoka Y. Epidemiology of Helicobacter pylori infection and public health implications. Helicobacter. 2011;16(Suppl 1):1-9.

7. Fedichkina TP, Solenova LG. [Helicobacter pylori: routes of transmission of infection (a review of literature)]. Gig Sanit. 20114):30-34.

8. Bures J, Kopacova M, Skodova Fendrichova M, Rejchrt S. [Epidemiology of Helicobacter pylori infection]. Vnitr Lek. 2011;57(12):993-999.

9. Muhsen K, Cohen D, Spungin-Bialik A, Shohat T. Seroprevalence, correlates and trends of Helicobacter pylori infection in the Israeli population. Epidemiol Infect. 2012;140(7):1207-1214.

10. Alvarado-Esquivel $\mathrm{C}$, Liesenfeld $\mathrm{O}$, Marquez-Conde JA, Cisneros-Camacho A, Estrada-Martinez S, Martinez-Garcia SA, Gonzalez-Herrera A, et al. Seroepidemiology of infection with Toxoplasma gondii in waste pickers and waste workers in Durango, Mexico. Zoonoses Public Health. 2008;55(6):306-312.

11. Alvarado-Esquivel C, Estrada-Martinez S, Pizarro-Villalobos H, Arce-Quinones M, Liesenfeld O, Dubey JP. Seroepidemiology of Toxoplasma gondii infection in general population in a northern Mexican city. J Parasitol. 2011;97(1):40-43.

12. Bronfman M, Guiscafre H, Castro V, Castro R, Gutierrez G. La medicion de la desigualdad: una estrategia metodologica, analisis de las caracteristicas socioeconomi- cas de la muestra. Arch Invest Med. 1988;19(4):351360 .

13. Torres J, Leal-Herrera Y, Perez-Perez G, Gomez A, Camorlinga-Ponce M, Cedillo-Rivera R, Tapia-Conyer $\mathrm{R}$, et al. A community-based seroepidemiologic study of Helicobacter pylori infection in Mexico. J Infect Dis. 1998;178(4):1089-1094.

14. Alvarado-Esquivel C. Seroepidemiology of Helicobacter pylori infection in a Mennonite community in Durango State, Mexico. Helicobacter. 2013;18(3):215221.

15. Sasidharan S, Lachumy SJ, Ravichandran M, Latha LY, Gegu SR. Epidemiology of Helicobacter pylori among multiracial community in Northern Peninsular, Malaysia: effect of age across race and gender. Asian Pac J Trop Med. 2011;4(1):72-75.

16. Patterson T, Straten E, Jimenez S. The prevalence of Helicobacter pylori antibody in different age groups in Central Texas. Clin Lab Sci. 2012;25(2):102-106.

17. Gao L, Weck MN, Stegmaier C, Rothenbacher D, Brenner H. Alcohol consumption, serum gamma-glutamyltransferase, and helicobacter pylori infection in a population-based study among 9733 older adults. Ann Epidemiol. 2010;20(2):122-128.

18. Naja F, Kreiger N, Sullivan T. Helicobacter pylori infection in Ontario: prevalence and risk factors. Can J Gastroenterol. 2007;21(8):501-506.

19. Kuepper-Nybelen J, Thefeld W, Rothenbacher D, Brenner H. Patterns of alcohol consumption and Helicobacter pylori infection: results of a population-based study from Germany among 6545 adults. Aliment Pharmacol Ther. 2005;21(1):57-64.

20. Ogihara A, Kikuchi S, Hasegawa A, Kurosawa M, Miki K, Kaneko E, Mizukoshi H. Relationship between Helicobacter pylori infection and smoking and drinking habits. J Gastroenterol Hepatol. 2000;15(3):271-276.

21. Zhang L, Eslick GD, Xia HH, Wu C, Phung N, Talley NJ. Relationship between alcohol consumption and active Helicobacter pylori infection. Alcohol Alcohol. 2010;45(1):89-94.

22. Zaterka S, Eisig JN, Chinzon D, Rothstein W. Factors related to Helicobacter pylori prevalence in an adult population in Brazil. Helicobacter. 2007;12(1):82-88.

23. Iso N, Matsuhisa T, Shimizu K. Helicobacter pylori Infection among patients visiting a clinic in Kasama City, Ibaraki Prefecture. J Nippon Med Sch. 2005;72(6):341354. 